\title{
Cardiofaciocutaneous syndrome
}

INSERM

\section{Source}

INSERM. (1999). Orphanet: an online rare disease and orphan drug data base.

Cardiofaciocutaneous syndrome. ORPHA:1340

Cardiofaciocutaneous (CFC) syndrome is a RASopathy characterized by craniofacial dysmorphology, congenital heart disease, dermatological abnormalities (most commonly hyperkeratotic skin and sparse, curly hair), growth retardation and intellectual disability. 